\title{
Some of My Favorite Problems in Ramsey Theory
}

\author{
Ron Graham *
}

December 7, 2006

\begin{abstract}
In this brief note, I will describe a variety of problems from Ramsey theory on which I would like to see progress made. To encourage this progress, I am offering modest rewards for most of these problems. This material is based on a 20-minute talk I gave at the meeting in Carrollton.
\end{abstract}

AMS Mathematics Subject Classification: 05D10

Keywords: Ramsey Theory

\section{The growth of $R(n)$}

Define $R(n)$ to be the least integer such that any graph on $R(n)$ vertices contains either a clique of size $n$ or an independent set of size $n$. One of the oldest open problems in Ramsey theory, raised by Erdös in the '30's, is to determine or at least estimate, the rate of growth of $R(n)$. Some of the earliest estimates (due to Erdős [8]) are:

$$
\frac{1}{e \sqrt{2}} n 2^{n / 2}(1+o(1))<R(n) \leq\left(\begin{array}{c}
2 n-2 \\
n-1
\end{array}\right)
$$

Unfortunately, very little progress has been made in the past half century on these bounds (and this is not for lack of trying!). The best bounds currently available for $R(n)$ (until very recently) were:

$$
\frac{2}{e \sqrt{2}} n 2^{n / 2}(1+o(1))<R(n)<n^{-1 / 2+c \sqrt{\log n}}\left(\begin{array}{c}
2 n-2 \\
n-1
\end{array}\right)
$$

*Department of Computer Science \& Engineering, University of California, San Diego. Research supported in part by Grant CCR-0310991. 
for a suitable $c>0$. The lower bound is due to Spencer [31] while the upper bound is due to Thomason [33]. However, a significant improvement on the upper bound was just established by Conlon [6]. He showed that for a suitable $c>0$,

$$
R(n+1)<\exp \left(-c\left(\frac{\log n}{\log \log n}\right)^{2} \log \log \log n\right)\left(\begin{array}{c}
2 n \\
n
\end{array}\right) .
$$

This implies that

$$
R(n+1)=O\left(\frac{1}{n^{s}}\left(\begin{array}{c}
2 n \\
n
\end{array}\right)\right)
$$

for every $s>0$.

Problem 1. (\$100) Prove that $\lim _{n \rightarrow \infty} R(n)^{1 / n}$ exists.

Problem 2. (\$250) Assuming this limit exists, what is it?

Of course, the limit would have to lie between $\sqrt{2}$ and 4 . One popular guess is that it is 2. (Well, why not!)

Both of these problems (and the associated prizes) were frequently mentioned by Erdös in his uncountably many talks and problems papers. More complete descriptions of these and many other related problems in this vein can be found in the monograph Erdös on Graphs: His Legacy of Unsolved Problems by F. Chung and the author [4].

Problem 3. (\$100) Give a constructive proof that for some $c>0$, $R(n)>(1+c)^{n}$.

As is well known, the best lower bounds for $R(n)$ are obtained using the probabilistic method [1].

\section{The Erdős/Szekeres Problem}

For each positive integer $n$, let $f(n)$ denote the least integer such that any set of $f(n)$ points in the plane in general position always contains a convex subset of size $n$. The general problem here is to determine or at least estimate the function $f(n)$.

This problem has a long and very interesting history which can be found, for example, in [21]. In particular, the original paper of Erdős and Szekeres 
[13] contained an independent proof of Ramsey's theorem, among other now classical results. Their original 1935 estimates

$$
2^{n-2}+1 \leq f(n) \leq\left(\begin{array}{c}
2 n-4 \\
n-2
\end{array}\right)+1
$$

remained unchanged until 1997, at which time the upper bound was improved by Fan Chung and the author [5] to:

$$
f(n) \leq\left(\begin{array}{c}
2 n-4 \\
n-2
\end{array}\right)
$$

a modest improvement, to be sure! However, this was followed by a rapid series of further improvements, the current best being that of Tóth and Valtr 34

$$
f(n) \leq\left(\begin{array}{c}
2 n-5 \\
n-2
\end{array}\right)+5
$$

which is about half as large as the original upper bound. It is suspected by many people that the lower bound is the truth. It certainly cannot be improved since it is shown in [14], that there are sets of $2^{n-2}$ points in the plane in general position which contain no convex subset of size $n$. (It is a nice exercise to construct such sets if you haven't already seen the construction). This prompts the following:

Problem 4. (\$1000) Prove or disprove that

$$
f(n)=2^{n-2}+1 .
$$

This is known to hold for $n \leq 5$.

As a warm-up, one might like to first solve

Problem 5. (\$100) Show that

$$
f(n)=o\left(\frac{4^{n}}{\sqrt{n}}\right)
$$

\section{Partition Regular Equations}

We say that an equation $f(x, y, z, \ldots)=0$ is partition regular if for any partition of the non-negative numbers $\mathbb{N}$ into finitely many classes $C_{1}, C_{2}, \ldots, C_{r}$, some $C_{i}$ contains a non-trivial solution to the equation. (Non-trivial means not all the variables are equal). Often we think of the $C_{i}$ as colors, and the solution in a single class as monochromatic. A rather complete theory of partition regularity for (systems of) of linear equations was developed by Rado [25]. For example, $x+y=z$ and $x+y=2 z$ are 
partition regular, but not $x+y=3 z$. In fact, a single homogenous equation over $\mathbb{N}$ is partition regular if and only if it has a non-trivial solution in 0's and 1's, (i.e., not all 0). However, for nonlinear equations, the situation is much less clear. For example, it was shown by Rödl [26] that the equation $1 / x+1 / y=1 / z$ is partition regular. Another partition regularity result for nonlinear equations is the striking result of Croot [7] who showed that the equation $\sum_{x \in S} 1 / x=1, S$ finite, is partition regular. In fact, he shows that an apppropriate $S$ can always be found in the interval $\left[2, e^{167000 r}\right]$ when $r$ colors are used. The following problem of Erdös and the author has been open for about 30 years:

Problem 6. (\$250) Determine whether the equation

$$
x^{2}+y^{2}=z^{2}
$$

is partition regular.

There is actually very little data (in either direction) to know which way to guess.

\section{The Chromatic Number of the Plane}

In this problem, which goes back to Nelson in 1950 (and perhaps even to Hadwiger in 1944; see [30, 3]), we are asked for the minimum number $r$ of colors needed so that the points of the Euclidean plane $\mathbb{E}^{2}$ can be $r$-colored in such a way that any two points separated by distance 1 have different colors. This number, called the chromatic number of the plane, and denoted by $\chi\left(\mathbb{E}^{2}\right)$, is known to satisfy

$$
4 \leq \chi\left(\mathbb{E}^{2}\right) \leq 7
$$

Both of these inequalities are quite easy to see, and have been known since the time the problem was suggested. If we operate in Zermelo-Fraenkel set theory with the Axiom of Choice (ZFC), then it follows by compactness that if $\chi\left(\mathbb{E}^{2}\right)=r$, then in fact there is a finite set which also requires $r$ colors to legally color it. However, as pointed out recently by Shelah and Soifer [29], if we replace the Axiom of Choice by several equally consistent axioms (Dependent Choice and every set is Lebesgue measurable), then we no longer have compactness and the answer can change. Also, O'Donnell [23], [24] has shown that for every integer $g$, there is a unit distance graph in $\mathbb{E}^{2}$ with girth greater than $g$ which has chromatic number 4 . Perhaps, this is evidence that $\chi\left(\mathbb{E}^{2}\right)$ is at least 5 ? 
Problem 7. (\$100) Show that $\chi\left(\mathbb{E}^{2}\right) \geq 5$.

Problem 8. (\$250) Show that $\chi\left(\mathbb{E}^{2}\right) \leq 6$.

In higher dimensions, it is known that (see [3]):

$$
6 \leq \chi\left(\mathbb{E}^{3}\right) \leq 15, \quad(1.239+o(1))^{n}<\chi\left(\mathbb{E}^{n}\right)<(3+o(1))^{n}
$$

\section{$5 \quad$ Euclidean Ramsey Sets}

Let us say that a finite subset $X$ of Euclidean space is Ramsey if for any number of colors $r$, there is an integer $N=N(X, r)$ such that in any $r$ coloring of the points of $\mathbb{E}^{N}$, there is always a monochromatic "copy" $X^{\prime}$ of $X$. In other words, $X^{\prime}$ can be obtained from $X$ by some Euclidean motion (rotation and translation). This subject had its genesis in a series of papers by Erdös et al. [10, 11, 12. In particular, it was shown that the Cartesian product of Ramsey sets is Ramsey, so that since a 2-point set is obviously Ramsey, then so is any (subset) of a rectangular parallelepiped. On the other hand, it was also shown that any Ramsey set $X$ must lie on the surface of some sphere. In such a case, we say that $X$ is spherical.

Problem 9. (\$1000) Prove that all spherical sets are Ramsey.

As a warm-up to this problem, one might work on the simpler:

Problem 10. (\$100) Prove that any 4-point subset of a circle is Ramsey.

In order not to be too discouraging, we mention several more (presumably easier) Euclidean Ramsey problems.

Conjecture 1. $(\$ 25)$ For any 3 -point set $\mathrm{T}$, there is a 3-coloring of $\mathbb{E}^{2}$ which has no monochromatic copy of $\mathrm{T}$.

Conjecture 2. (\$50) In any 2-coloring of $\mathbb{E}^{2}$, a monochromatic copy of every 3-point set occurs, except possibly for a single equilateral triangle. 
Fact. For any set $L$ of 3 collinear points, there is a 16 -coloring of $\mathbb{E}^{n}$ which contains no monochromatic copy of $L$.

Question. Is 16 the best possible constant here?

Perhaps the right answer is 4 . (or even 3 !).

\section{Van der Waerden's Theorem}

The classical theorem of van der Waerden [35] on arithmetic progressions asserts that for any finite coloring of $\mathbb{N}$, there always exists arbitrarily long monochromatic arithmetic progressions. The finite version (for two colors) guarantees the existence of a least number $W(n)$ such that if the integers $\{1,2, \ldots, W(n)\}$ are 2 -colored, then a monochromatic $n$-term arithmetic progression ( $n$-AP) must always be formed. The estimation of the function $W(n)$ has challenged mathematicians ever since van der Waerden proved this result in 1927. The first upper bound, due to van der Waerden, grew like the Ackermann function, and was not even primitive recursive (his proof was a double induction on $n$ and the number of colors). This was finally remedied by a new proof by Shelah [28] who reduced it to a function residing in the 5th level of the Gregorchik hierarchy (basically towers of towers). The current champion is based on a sensational result of Gowers [17] concerning the upper density of subsets of $[1, N]$ which contain no $k$-AP. From this result, one can deduce the estimate that for all $n$, we have:

$$
W(n)<2^{2^{2^{2^{n+9}}}}
$$

In particular, this settled a long-standing conjecture I had made on the size of $W(n)$ (which asserted that $W(n)$ was upper-bounded by an exponential tower of 2's of height $n$ ), and as a result, left me $\$ 1000$ poorer (but much happier). Undaunted, I now propose the following:

Conjecture. (\$1000) For all $n$,

$$
W(n)<2^{n^{2}}
$$

I might point out that the best lower bound (due to Berlekamp [2] has been around for almost 40 years:

$$
W(n+1) \geq n 2^{n}
$$

for $n$ prime (the proof uses finite fields).

\section{Isn't it about time for some improvement here?}




\section{Combinatorial Lines}

For a finite set $A=\left\{a_{1}, a_{2}, \ldots, a_{t}\right\}$, let $A^{N}$ denote the set of $N$-tuples from $A$. A combinatorial line in $A^{N}$ is a set of $t N$-tuples $X_{1}, X_{2}, \ldots, X_{t}$ where

$$
X_{k}=\left(X_{k}(1), X_{k}(2), \ldots, X_{k}(N)\right)
$$

and for $1 \leq j \leq N$, either all $X_{k}(j)$ are equal, or $X_{k}(j)=a_{k}, 1 \leq k \leq t$. This concept was first introduced in the seminal paper of Hales and Jewett [22].

In 1990, Furstenberg and Katznelson [16] proved the following beautiful theorem, generalizing Szemerédi's great density theorem for arithmetic progressions [32]:

Theorem. For every $\epsilon>0$, there exists a least $N=N(\epsilon, t)$ such if $R \subseteq A^{N}$ with $|R|>\epsilon t^{N}$ then $R$ must contain a combinatorial line.

Unfortunately, the ergodic theory tools used by Furstenberg and Katznelson do not allow us to conclude anything about the growth rate of $N(\epsilon, t)$ as $\epsilon \rightarrow 0$.

Problem. Establish any upper bound on $N(\epsilon, t)$.

The case of $t=2$ is instructive (and is the only case we can handle!). In this case, using $A=\{0,1\}$, we see that a combinatorial line in $A^{N}$ is equivalent to having two subsets $X, Y \subseteq\{1,2, \ldots, N\}$ with $X \subset Y$. By the well known result of Sperner, this must happen as soon as $|R|>\left(\begin{array}{c}N \\ \lfloor N / 2\rfloor\end{array}\right)$. This implies that $N(\epsilon, 2)<c \epsilon^{-2}$ for a suitable $c>0$.

Warm-up Problem. Establish an upper bound on $N(\epsilon, 3)$.

In particular, it would be of great interest to obtain Gowers' type bounds on these quantities, that is, bounded towers of exponents (which might be called "Gowers towers"!).

\section{Concluding Remarks}

Of course, in this brief note I have only been able to touch on a few of the problems in this area that are most attractive to me, and for which I feel the time is ripe for making further progress. Much richer collections of problems and results in this subject can be found in a variety of sources, such as [3], [5], [19], [18], and [20]. 


\section{References}

[1] Noga Alon and Joel Spencer, The Probabilistic Method, Second Edition, Wiley-Interscience [John Wiley \& Sons], New York, (2000). xviii+301 pp.

[2] E. R. Berlekamp, A construction for partitions which avoid long arithmetic progressions, Canad. Math. Bull. 11 (1968), 409-414.

[3] P. Brass, W. Moser and J. Pach, Research Problems in Discrete Geometry Springer, New York, (2005). xii+499 pp.

[4] Fan Chung and Ron Graham, Erdős on Graphs: His Legacy of Unsolved Problems, A K Peters, Ltd., Wellesley, MA, (1998). xiv+142 pp.

[5] F. R. K. Chung and R. L. Graham, Forced convex $n$-gons in the plane, Discrete Comput. Geom. 19 (1998), 367-371.

[6] D. Conlon, New upper bounds for some Ramsey numbers (2006) (preprint), $34 \mathrm{pp}$.

[7] E. Croot, On a coloring conjecture about unit fractions. Ann. of Math. (2) 157 (2003), 545-556.

[8] P. Erdös, Some remarks on the theory of graphs, Bull. Amer. Math. Soc. 53 (1947), 292-294.

[9] P. Erdös and R. L. Graham, Old and New Problems and Results in Combinatorial Number Theory, Monographies de L'Enseignement Mathématique, 28, Université de Genève, Geneva, (1980) $128 \mathrm{pp}$.

[10] P. Erdős, R. L. Graham, P. Montgomery, B. L. Rothschild, J. H. Spencer, and E. G. Straus, Euclidean Ramsey Theorems. I J. Combin. Theory Ser. A 14 (1973), 341-363.

[11] P. Erdős, R. L. Graham, P. Montgomery, B. L. Rothschild, J. H. Spencer, and E. G. Straus, Euclidean Ramsey Theorems. II. Infinite and finite sets (Colloq., Keszthely, 1973, Vol I, pp. 529-557. Colloq. Math. Soc. Janos Bolyai, Vol. 10, North-Holland, Amsterdam, (1975).

[12] P. Erdős, R. L. Graham, P. Montgomery, B. L. Rothschild, J. H. Spencer, and E. G. Straus, Euclidean Ramsey Theorems. III. Infinite and finite sets (Colloq., Keszthely, 1973, Vol I, pp. 559-583. Colloq. Math. Soc. Janos Bolyai, Vol. 10, North-Holland, Amsterdam, (1975). 
[13] P. Erdős and G. Szekeres, A combinatorial problem in geometry, Composito Math. 2 (1935), 464-470.

[14] P. Erdős and G. Szekeres, On some extremum problems in elementary geometry. Ann. Univ. Sci. Budaest. Eőtvős Sect. Math. 3-4 (19601961), 53-62.

[15] P. Erdős and P. Turán, On some sequences of integers, J. London Math. Soc. 11 (1936), 261-264.

[16] H. Fürstenberg, and Y. Katznelson, An ergodic Szemerédi theorem for commuting transformations, J. Analyse Math. 34 (1979), 275-291.

[17] W. T. Gowers, A new proof of Szemerédi's theorem, Geom. Funct. Anal. 11 (2001), 465-588.

[18] W. T. Gowers, Some unsolved problems in additive/combinatorial number theory, (on Gowers Website at www.dpmms.cam.ac.uk/ ${ }^{\sim}$ wtg10/papers.html)

[19] R. L. Graham, Rudiments of Ramsey Theory. CBMS Regional Conference Series in Mathematics, 45. American Mathematical Society, Providence, R.I., (1981). v+65pp.

[20] R. L. Graham, B. L. Rothschild, and J. H. Spencer, Ramsey Theory Second edition. John Wiley $\&$ Sons, Inc., New York, (1990). xii+196 pp.

[21] R. L. Graham and J. Nešetřil, Ramsey Theory and Paul Erdős (recent results from a historical perspective). Paul Erdös and his mathematics, II (Budapest, 1999), 339-365, Bolyai Soc. Math. Stud., 11, Já nos Bolyai Math. Soc., Budapest, (2002)

[22] A. W. Hales and R. I Jewett, Regularity and positional games, Trans. Amer. Math. Soc. 106 (1963), 222-229.

[23] P. O'Donnell, Arbitrary girth, 4-chromatic unit distance graphs in the plane. I. Graph embedding. Geombinatorics 9 (2000), 180-193.

[24] P. O'Donnell, Arbitrary girth, 4-chromatic unit distance graphs in the plane. II. Graph description. Geombinatorics 9 (2000), 145-152.

[25] R. Rado, Studien zur Kombinatorik, Math. Zeit. 36 (1933), 242-280.

[26] V. Rődl, (personal communication).

[27] K. F. Roth, On certain sets of integers, J. London Math. Soc. 28 (1953), 104-109. 
[28] S. Shelah, Primitive recursive bounds for van der Waerden numbers, J. Amer. Math. Soc. 1 (1988), 683-697.

[29] S. Shelah and A. Soifer, Axiom of choice and chromatic number: examples on the plane. J. Combin. Theory Ser. A 105 (2004), 359-364.

[30] A. Soifer, Chromatic number of the plane: its past and future. Proceedins of the Thirty-Fourth Southeastern International Conference on Combinatorics, Graph Theory and Computing. Congr. Numer. 160, (2003), 69-82.

[31] J. H. Spencer, Ramsey's Theorem-A New Lower Bound, J. Comb. Th. (A) 18 (1975), 108-115.

[32] E. Szemerédi, On sets of integers containing no $k$ elements in arithmetic progression, Acta Arith. 27 (1975), 199-245.

[33] A. Thomason, An upper bound for some Ramsey numbers, J. Graph Theory 12 (1988), no. 4, 509-517.

[34] G. Tóth and P. Valtr, Note on the Erdős-Szekeres theorem, Discrete Comput. Geom. 19 (1998), 457-459.

[35] B. L. van der Waerden, Beweis einer Baudetschen Vermutung, Nieuw Arch. Wiskunde 15 (1927), 212-216. 